(C) Journal of Applied Mathematics \& Decision Sciences, 4(2), 151-163 (2000)

Reprints Available directly from the Editor. Printed in New Zealand.

\title{
Comparison Theorems for Multicomponent Diffusion Systems: Developments since 1961
}

M.I. NELSON

amt5man@amsta.leeds.ac.uk

Department of Fuel and Energy, The University of Leeds, Leeds LS2 9JT, UK.

\begin{abstract}
Comparison theorems may be used to prove the existence and uniqueness of solutions to certain types of partial differential equations. They provide bounds for solutions and can be used as the basis of numerical techniques for the computation of solutions. In 1961 Alex McNabb published one of the first papers extending their use to multi-component systems. Developments in the theory and applications of such results, through citations of this original paper, are reviewed.
\end{abstract}

Keywords: comparison theorems, reaction-diffusion equations.

\section{Introduction}

For a scalar nonlinear diffusion equation the maximum principle and comparison theorems lead to existence and uniqueness results for initial-boundary-value problems by supplying a priori bounds on the solution. They are also a useful tool in investigating solution stability.

There has been a considerable amount of activity over the last thirty years in extending these methods to certain systems of parabolic partial differential equations. In general there are limitations when considering multi-component systems, which leads to weaker results than is the case for the scalar equation.

Maximum principles are discussed in detail in Protter and Weinberger[21] and Sperb[23], and for parabolic equations in Friedman[6]. A good general introduction to the theory of systems of reaction-diffusion equations is provided by Smoller[22]. The applications of the maximum principle and comparison theorems to mathematical biology is covered by Britton[1].

One of the first English-language papers to extend these techniques to multicomponent systems was by McNabb[12]. We review developments in the theory and applications of multi-component comparison systems as revealed through citations of this paper.

For the sake of brevity the full requirements to prove the results summarised in this paper are not provided. Similarly, technical extensions of the results are not included. The interested reader is referred to the original papers for these details. 


\section{Alex McNabb's 1961 paper and its immediate extensions}

Let $B$ denote a bounded $n$-dimensional domain of real variables $(\mathbf{x})=\left(x_{1}, \ldots, x_{n}\right)$ and $D_{T}$ the product space $\{(\mathbf{x}, t): \mathbf{x} \in B, 0<t \leq T\} . \bar{B}$ and $\overline{D_{T}}$ are the closures of $B$ and $D_{T}, \partial B$ is the boundary of $B$, and $S_{T}$ is the cylinder $\{(\mathbf{x}, t): \mathbf{x} \in \partial B, 0<t \leq T\}$. $\operatorname{In}[12] \mathrm{McNabb}$ considered the nonlinear parabolic system

$$
\begin{aligned}
L(u) & \equiv \sum_{i, j=1}^{n} a_{i j}(\mathbf{x}, t) \frac{\partial^{2} u}{\partial x_{i} \partial x_{j}}+\sum_{i=1}^{n} b_{i}(\mathbf{x}, t) \frac{\partial u}{\partial x_{i}}+c(\mathbf{x}, t) u-\frac{\partial u}{\partial t}, \\
L(u) & =f(\mathbf{x}, t, u, w), \\
\frac{\partial w}{\partial t} & =g(\mathbf{x}, t, u, w),
\end{aligned}
$$

with coefficients defined in $\overline{D_{T}}$ and functions $f, g$ defined for all values of the variables $(\mathbf{x}, t, u, w)$ in a region $R$ of $(\mathbf{x}, t, u, w)$ space. In this space $f$ is a nonincreasing function of $w, g$ a nondecreasing function of $u$ and both satisfy a uniform Lipschitz condition

$$
\left|h\left(\mathbf{x}, t, u_{1}, w_{1}\right)-h\left(\mathbf{x}, t, u_{2}, w_{2}\right)\right| \leq M\left(\left|u_{1}-u_{2}\right|+\left|w_{1}-w_{2}\right|\right),
$$

where $(\mathbf{x}, t) \in \overline{D_{T}},\left(\mathbf{x}, t, u_{1}, w_{1}\right)$ and $\left(\mathbf{x}, t, u_{2}, w_{2}\right) \in R$ and $h \equiv f$ or $g$.

McNabbs' interest in this system stemmed from the diffusion of hydrogen in steel (private communication). "The problem was geothermal in origin: would there be embrittlement problems with turbine blades because of hydrogen sulphide in geothermal steam? Analysis based purely on diffusion was in trouble as estimated diffusivities varied by orders of magnitude from one steel to another. We tracked down the difficulty to hydrogen trapping: work hardening generated dislocation sites in the steel which trapped hydrogen. The new theory involved diffusing hydrogen and an immobile trapped phase. There was a diffusion equation for the mobile phase coupled to a reaction equation for hydrogen with the trapping sites. This is a nonlinear first order equation. The problem was too difficult to solve analytically and so approximation techniques were needed. Having used comparison theorems for the spontaneous combustion of wool our first thoughts were, could the same technique be used for this coupled diffusion problem? This was about the simplest extension to the one dimension theory you could imagine and it worked. Basically the reaction equation was monotone in the coupling variables. Once you see this success is due to monotonicity the extension to systems of monotonically coupled equations was obvious."

Theorem 1 (Weak Comparison Theorem[12]) Suppose,

(a) $u_{1}, u_{2}, w_{1}, w_{2}$ exist and are continuous in $\overline{D_{T}}$.

(b) Their second order $x_{i}$-derivatives and first order $t$-derivatives exist and are uniformly bounded in $D_{T}$, satisfying their the inequalities 


$$
\begin{aligned}
& L\left(u_{1}\right)-f\left(\mathbf{x}, t, u_{1}, w_{1}\right) \geq L\left(u_{2}\right)-f\left(\mathbf{x}, t, u_{2}, w_{2}\right), \\
& \frac{\partial w_{1}}{\partial t}-g\left(\mathbf{x}, t, u_{1}, w_{1}\right) \leq \frac{\partial w_{2}}{\partial t}-g\left(\mathbf{x}, t, u_{2}, w_{2}\right) . \\
& \text { (c) } u_{1} \leq u_{2} \text { on } S_{T} \text { and } u_{1} \leq u_{2}, w_{1} \leq w_{2} \text { on } \bar{B} \text { at } t=0 . \\
& \text { Then } u_{1} \leq u_{2} \text { and } w_{1} \leq w_{2} \text { in } \overline{D_{T}} .
\end{aligned}
$$

The functions pairs defined by conditions (a) and (b) in this theorem also satisfy strong comparison theorems. These results readily lead to uniqueness proofs for the Dirichlet and related problems. The comparison theorems are then used to prove the existence of solutions of equations (2) \& (3). Similar results hold when $u$ and $w$ are vectors. The Cauchy problem associated with equations (2-3) was considered by Kusano[9]. He proved a strong comparison theorem, again leading to a uniqueness result, and an existence theorem.

Mo[15] considers the Dirichlet problem for a singularly perturbed reaction diffusion system

$$
\begin{aligned}
\frac{\partial u_{i}}{\partial t}-\left(\epsilon L^{i}+L_{1}^{i}\right) u_{1} & =f_{i}\left(t, x, u_{r}, w_{s}, \epsilon\right), i=1, \ldots, N_{1} \\
\frac{\partial w_{i}}{\partial t} & =g_{i}\left(t, x, u_{r}, w_{s}, \epsilon\right), i=1, \ldots, N_{2},
\end{aligned}
$$

where $\epsilon$ is a small positive parameter, $L^{i}$ is a second-order uniform-elliptic operator, and $L_{1}^{i}$ is a first order partial differential operator. A uniformly valid asymptotic solution, to any degree of precision, is obtained by combining the method of variable scales with comparison theorems.

Theorem 1 requires rather restrictive conditions on the nonlinear functions. One of the main aims in developments of this work is the derivation of comparison theorems with less restrictive restraints. However, a comparison theorem is impossible for a general multicomponent diffusion system without some restrictions on the reaction functions[24]. $\operatorname{In}[24]$ a counter-example is constructed in which, although requirement (b) of Theorem 1 is satisfied, the supposed comparison functions are not consistent upper and lower bounds for the solution.

\section{Applications to chemical systems}

\subsection{The slow combustion problem with an immobilised reactant}

Wake[25] considers the initial-value problem consisting of equations (2-3) with Dirichlet boundary conditions. Equation (2) is the heat conduction equation for the temperature $u(\mathbf{x}, t)$ with heat sources dependent on the reactant concentration $w(\mathbf{x}, t)$ as well as the temperature, position, and time. The reactant concentration is governed by equation (3). Uniqueness and existence is established assuming that $f$ and $g$ satisfy the uniform Lipschitz condition (4). Monotonicity requirements 
are not imposed on these functions. Existence is proved by the construction of a subsolution and a supersolution combined with a strong comparison theorem.

The functions $f$ and $g$ are then specified by taking an Arrhenius formulation

$$
f=g= \begin{cases}-w^{m} \exp [-k / u] & (w>0), \quad k, m>0, \\ 0 & (w \leq 0) .\end{cases}
$$

In a neighbourhood of the points where $w=0$, the functions $f$ and $g$ do not satisfy the Lipschitz condition unless $m \geq 1$. From a practical perspective the points of Lipschitz discontinuity coincide with exhaustion of the reactant. When $0 \leq m<1$ it follows from equation (3) that $w$ becomes zero in finite time. The problem then becomes one involving a moving boundary containing the unburnt material.

Wake generalises his existence and uniqueness theorems to systems where the functions $f$ and $g$ do not satisfy the Lipschitz condition (4) in the neighbourhood of $w=0$, yet remained bounded uniformly in $\bar{D}_{T}$. This is achieved by redefining them on the interval $0 \leq w \leq \epsilon$, so that the Lipschitz condition is satisfied in the neighbourhood of $w=0$.

Maslennikova and Gapeeva[11] consider the problem defined by equations (2-3) with the usual monotonicity requirements and the uniform Lipschitz condition (4). The boundary condition is

$$
\left.l u\right|_{S_{T}} \equiv \frac{\partial u}{\partial \vec{l}}+\left.b(\mathbf{x}, t) u(\mathbf{x}, t)\right|_{S_{T}} \equiv \sum_{k=1}^{n} a_{k}(\mathbf{x}, t) \frac{\partial u}{\partial x_{i}}+\left.b(\mathbf{x}, t) u\right|_{S_{T}}=\phi(\mathbf{x}, t)
$$

It is assumed that the condition

$$
\sum_{k=1}^{n} a_{k}(\mathbf{x}, t) \cos \left(\vec{n}, x_{k}\right) \geq a_{0}>0, a_{0}=\mathrm{const},(\mathbf{x}, t) \in S_{T},
$$

holds, where $\vec{n}$ is the inner normal at $(\mathbf{x}, t) \in S_{t}, S_{t}$ the intersection of the surface $S_{T}$ with the plane $t=$ const. Condition (9) implies that a directional derivative appears in the left-hand side of boundary condition (8) which is along a direction $\vec{l}$ lying in the plane $t=$ const and forming an acute angle with the inner normal $\vec{n}$ to $S_{t}$.

Systems of this form are described as modeling the absorbtion of a gas by a solution containing a chemical with which the gas reacts.

Weak and strong comparison theorems are proved which immediately imply a uniqueness theorem for this problem. Convergence of the method of successive approximations is also proved. (This is a constructive method in which monotone sequences of upper and lower solutions are obtained by iterating suitable initial functions. Under certain conditions the iteratitive solution converges to the solution of the problem. Such methods can be used to obtain a solution computationally.) These results also hold for the Cauchy problem, in which case it is assumed that the solution is bounded at infinity. 
A thermodynamic formulation for the thermochemical response of a rigid, bounded, regular body in Euclidean 3 -space is[3]

Constitutive Relations

$$
\begin{aligned}
e= & \hat{e}(\theta, \xi), \\
\mathbf{q}= & \hat{\mathbf{q}}(\theta, \xi, \nabla \theta), \\
\omega= & \hat{\omega}(\theta, \xi, \nabla \theta) \\
& \text { Reaction-diffusion equations on } B \times \Re \\
& \text { A system of } N \text { chemical species } \\
\dot{\xi}= & \omega, \\
& \text { Temperature equation } \\
\dot{e}= & \nabla \cdot \mathbf{q}+r,
\end{aligned}
$$

where $e$ denotes internal energy, $\mathbf{q}$ the heat flux vector, $\omega$ the reaction rate vector, $\theta$ the absolute temperature, $\xi$ the extent of reaction vector whose component $\xi_{i}, i=$ $1, \ldots, M$, represents the degree of advancement of the $i$ th chemical reaction, and $r$ the heat supply. The functions $\hat{e}, \hat{\mathbf{q}}, \hat{\omega}$ are assumed to be sufficiently differentiable.

Chen and Nunziato[4] assume that a state of chemical equilibrium exists and consider small departures from such a state. They show that the linearized initial boundary value problem has a unique solution. Chenet al[3] generalize Chen and Nunziato's observations and establish uniqueness under weaker conditions for the general nonlinear mixed problem using the method of energy integrals.

This system is essentially that studied by McNabb[12] and Wake[25], without monotonicity requirements imposed on the reaction rate vector and heat release.

\subsection{Reaction-diffusion systems in chemical reactors}

Pao[17] considers a 1-dimensional heat and mass transfer model with homogeneous Robin boundary conditions and initial conditions satisfying the boundary conditions:

$$
\begin{aligned}
& \text { Reaction-diffusion equations on } t>0 \text { and } 0<x<l \\
u_{t}= & a_{1}(x, t) u_{x x}+b_{1}(x, t) u_{x}+c_{1}(x, t) u+f_{1}(x, t, u, w) \\
w_{t}= & a_{2}(x, t) w_{x x}+b_{2}(x, t) w_{x}+c_{2}(x, t) w+f_{2}(x, t, u, w)
\end{aligned}
$$

where $a_{i}(x, t) \geq a_{0}>0$, for some positive constant $a_{0}$ and $c_{i}(x, t) \leq 0$.

Restriction are placed on the nonlinear functions $f_{i}$, motivated by the form of the functions appearing in tubular reactor models $\left(f_{1}=d_{1}(1-w) \exp \left[\frac{u}{1+\gamma u}\right]+h_{1}, f_{2}=\right.$ $\left.d_{2}(1-w) \exp \left[\frac{u}{1+\gamma u}\right]\right)$ : 
HyPOTHESIS 1 There exist positive constants $r, \rho_{1}$, and $\rho_{2}$ such that for each $t>0$ and $0 \leq x \leq 1$

$$
\begin{aligned}
& f_{1}(x, t, u, w) \geq 0 \text { if } u \geq 0 \text { and } 0 \leq w \leq r \\
& f_{2}(x, t, u, w) \geq 0 \text { if } u \geq 0 \text { and }-\infty \leq w \leq 0, \\
& f_{2}(x, t, u, w) \geq 0 \text { if } u \geq 0 \text { and } r \leq w \leq \infty
\end{aligned}
$$

and

$$
\left|f_{i}\left(x, t, u_{1}, w_{1}\right)-f_{i}\left(x, t, u_{2}, v_{2}\right)\right| \leq \rho_{i}\left(\left|u_{1}-u_{2}\right|+\left|w_{1}-w_{2}\right|\right)(i=1,2)
$$

for

$$
u_{1}, u_{2} \geq 0,0 \leq w_{1}, w_{2} \leq r
$$

Pao shows that under Hypothesis 1 there is a unique positive solution. Under additional assumptions on $f_{1}$ and $c_{1}$ it is shown that $u(x, t)$ is bounded, decaying exponentially with time to a constant. ( $w(x, t)$ is uniformly bounded by $r)$.

Existence is proved using the method of successive approximations. This requires that the functions $f_{i}$ satisfy some global Lipschitz conditions that are not necessarily fulfilled by functions satisfying Hypothesis 1 . To overcome this difficulty the functions $f_{i}$ are redefined $\left(\hat{f}_{i}\right)$. The modified problem (with $f_{i}$ replaced by $\hat{f}_{i}$ ) satisfies the conditions for the existence proof and has a unique solution. It turns out that the solution to the modified problem is a solution to the initial problem if the initial conditions satisfy $\phi_{1}(x) \geq 0$ and $0 \leq \phi_{2}(x) \leq r$.

In Theorem 1 equation (16) is replaced by equation (3) and Dirichlet condition conditions are used. Pao's results can be applied to systems of this form with less restrictions on the nonlinear functions.

$\operatorname{In}[19]$ Pao considers a general formulation for coupled reaction-diffusion systems arising from chemical diffusion processes and combustion theory with a single irreversible reaction in a porous medium.

Reaction-diffusion equations for $t>0$ and $\mathbf{x} \in B$.

$$
\begin{aligned}
u_{t}= & \nabla \cdot\left(D_{2}(\mathbf{x}) \nabla u\right)+b w^{m} f(\mathbf{x}, u), \\
w_{t}= & \nabla \cdot\left(D_{1}(\mathbf{x}) \nabla w\right)-a w^{m} f(\mathbf{x}, u), \\
& \text { Boundary Conditions on } t>0, \mathbf{x} \in \partial B \\
B_{2}[u]= & \alpha_{2}(\mathbf{x}) \frac{\partial u}{\partial v}+\beta_{2}(\mathbf{x}) u=h_{2}(x) \\
B_{1}[w]= & \alpha_{1}(\mathbf{x}) \frac{\partial w}{\partial v}+\beta_{1}(\mathbf{x}) w=h_{1}(x)
\end{aligned}
$$

Here $m \geq 1, a>0, b>0$ are constants, $D_{i}>0, \alpha_{i} \geq 0, \beta_{i} \geq 0$ with $\alpha_{i}+\beta_{i}>0$ on $\partial B, i=1,2, h_{1}, h_{2}$ are nonnegative functions on $\partial B$ and $\frac{\partial}{\partial v}$ is the outward normal derivative on $\partial B$. 
Various problems considered in the literature can be obtained from this formulation by suitable choice of the rate constant $f$ and the boundary conditions. The choice $D_{1} \equiv 0$ recovers the immobilised reactant problem discussed in section 3.1.

The following assumptions are made on the function $f$.

Hypothesis $2 \frac{\partial f}{\partial v}$ exists and is bounded on bounded subsets of $\bar{B} \times \Re^{+}$; and there exists a function $c_{0}(\mathbf{x}) \geq 0$ such that

$$
0 \leq f\left(\mathbf{x}, u_{1}\right) \leq f\left(\mathbf{x}, u_{2}\right) \leq c_{0}(\mathbf{x}) \quad \text { for } 0 \leq u_{1} \leq u_{2}<\infty .
$$

Condition (26) implies that $f$ is monotone nondecreasing in $u$ and is uniformly bounded for $u \geq 0$. Thus the functions

$$
\begin{aligned}
& F_{1}(\mathbf{x}, u, w)=-a w^{m} f(\mathbf{x}, w), \\
& F_{2}(\mathbf{x}, u, w)=b w^{m} f(\mathbf{x}, w),
\end{aligned}
$$

are quasi-monotone nonincreasing and quasi-monotone nondecreasing respectively.

Qualitative properties of the solution are investigated using comparison methods and the notion of sub- and super-solutions. This analysis includes the existence and uniqueness of positive time-dependent solutions, lower and upper bounds of the solution, asymptotic behaviour and invariant sets of the solution, stability of steady-state solutions, including an estimate of the stability region. Explicit conditions for the asymptotic behaviour and the stability of a steady-state solution are given. Existence is proved by applying the method of successive approximations to a sub- and super-solution. The usefulness of this existence-comparison theorem, which remains true when one of the diffusion coefficients is zero, is that suitable construction of sub- and super-solutions not only ensures existence, but enables the stability problem and the asymptotic behaviour of the time-dependent solution to be established from the behaviour of the sub- and super-solutions.

Special attention is given to the role played by the boundary conditions since under the same set of physical parameters and reaction function, solutions to the system with Neumann, Dirichlet or Robin boundary conditions exhibit quite different asymptotic behaviour. For instance, under pure Neumann boundary conditions $\left(\beta_{i}=h_{1}=0, i=1,2\right)$ the solution $(u, w)$ of equations (22-25) converges to $\left(\hat{u}_{0}+\frac{b}{a} \hat{w}_{0}, 0\right)$ as $t \rightarrow \infty$ when $w_{0} \neq 0$ and to $\left(\hat{u}_{0}, 0\right)$ when $w_{0}=0$, where $\hat{u}_{0}, \hat{w}_{0}$ are the spatial averages of the initial conditions $u_{0}, w_{0}$. On the other hand, if the boundary condition is of Dirichlet or Robin with $h_{1}=h_{2}=0$, then under the same set of physical parameters, the solution always converges (in exponential order) to $(0,0)$. In the general nonhomogeneous system where $h_{1}, h_{2}$ are not both zero, a sufficient condition for ensuring the global asymptotic stability of a steady-state solution is given. A direct consequence of this result is that by increasing the diffusion coefficients or decreasing the size of the diffusion medium the general system has exactly one steady-state solution which is globally asymptotically stable.

This paper is a tour-de-force in the repeated construction of sub- and supersolutions followed by applications of comparison theorems. 
Parshotam et al[20] prove comparison theorems for a general system of reactiondiffusion equations involving interacting macro and microstructures where concentrations in the macrosystem determine the boundary conditions of the microsystem which in turn governs source strengths for components in the macrosystem. Such systems arise as models for many types of reactor beds used in industrial processes.

The model consists of a system of reaction-diffusion equations coupled to convective reaction-diffusion equations via the boundary conditions and a source term. The overall system consists of weakly coupled, degenerate, parabolic equations. The proof of the multi-component comparison theorems require a strong comparison theorem of elliptic type for a scalar equation. Comparison theorems are used to deduce uniqueness and to provide stability results. The latter require that the reaction functions satisfy certain monotonicity requirements. If these are not satisfied it is shown that the system can be embedded into a new system of twice the size satisfying these requirements and that existence, uniqueness, and stability results for the enlarged system imply existence, uniqueness, and stability for the original system.

\section{Applications to biological systems}

Williams and Chow[26] consider nonlinear reaction-diffusion equations modelling the population dynamics of M species satisfying no-flux boundary conditions

$$
\frac{\partial c_{i}}{\partial t}=\nu_{i}(\mathbf{x}, t) \Delta c_{i}+c_{i} B_{i}\left(c_{1}, \ldots, c_{M}\right), \quad 1 \leq i \leq M,
$$

where the diffusion coefficients are bounded by $\nu_{i} \geq \delta>0$.

The functions $B_{i}$ are restricted by imposing a 'food pyramid condition'. This condition means that it is possible to arrange the species in numerical sequence so that if $1 \leq i<j \leq M$, then the $i$ th species does not use the $j$ th species for food. If $c_{1} \leq P, \ldots, c_{i-1} \leq P$, then all the species that the $i$ th species might use for food (except the $i$ th species itself) have limited concentrations, and thus (since cannibalism does not increase the biomass of the $i$ th species) its growth is limited, i.e. $B_{i}\left(c_{1}, \ldots, c_{M}\right) \leq b_{i}(P)$. The first species, and perhaps a few others, is a primary food producer, not feeding on any other species. Its growth rate is restricted by the limited amount of energy density in the environment so it is reasonable to assume that there is a $b_{1}>0$ such that $B_{1}\left(c_{1}, \ldots, c_{M}\right)<b_{1}$ whenever $c_{1} \geq 0, \ldots, c_{M} \geq 0$.

The authors prove that the solution of this system exists for all positive time and is unique, even among generalised solutions, smooth, and strictly positive for $t>0$. Thus, unlike the situation without diffusion, no species can be driven to extinction. 
Capasso[2] considers a reaction-diffusion system involving an integrodifferential linear parabolic equation and a nonlinear ordinary differential equation

$$
\begin{aligned}
\frac{\partial}{\partial \mathrm{t}} u(\mathbf{x}, t) & =d_{1} \Delta u(\mathbf{x}, t)-a_{11} u(\mathbf{x}, t)+\int_{B} k\left(\mathbf{x}, \mathbf{x}^{\prime}\right) w\left(\mathbf{x}^{\prime}, t\right) \mathrm{d} \mathbf{x}^{\prime}, \\
\frac{\partial}{\partial \mathrm{t}} w(\mathbf{x}, t) & =-a_{22} w(\mathbf{x}, t)+g(u(\mathbf{x}, t)),
\end{aligned}
$$

Robin Boundary Condition on $(\mathbf{x}, t) \in \partial B \times(0,+\infty)$.

$$
0=\beta(\mathbf{x}) \frac{\partial}{\partial v} u(\mathbf{x}, t)+\alpha(\mathbf{x}) u(\mathbf{x}, t)
$$

where $a_{11}>0, a_{12}>0, \frac{\partial}{\partial v}$ denotes the outward normal derivative on $\partial B$ and $\alpha(\mathbf{x})+\beta(\mathbf{x})>0, \mathbf{x} \in \partial B$. Suitable restrictions are placed on the kernel $k\left(\mathbf{x}, \mathbf{x}^{\prime}\right)$. The function $g \in \mathcal{C}^{2}\left(\Re^{+} \rightarrow \Re^{+}\right)$is strictly monotonic increasing with $g(0)=0$, $g_{+}^{\prime}(0)$ exists and $g^{\prime \prime}(z)<0$ for $0<z$.

This system describes the evolution of a class of man-environment epidemic phenomena; in particular applying to to man-environment interactions due to either pollutants or infectious agents in the environment which are fed by human or other populations. The integral term takes into account distant interaction between the two involved species.

A comparison theorem is outlined which proves existence of a unique solution for all time. Comparison theorem techniques are used to find the conditions under which the trivial solution is either globally asymptotically stable or unstable. Monotone iteration techniques are combined with a strong comparison theorem to show that when the trivial solution is unstable there is a unique nontrivial equilibrium solution which is globally asymptotically stable.

McNabb \& Bass[14] consider a reaction-diffusion model for the cellular uptake of protein-bound ligands. Biological structures (cells) of arbitrary shapes are suspended in a liquid (plasma) containing small molecules (ligand) bound reversibly to large molecules (protein). The resulting three molecular species all diffuse, but only the free ligand is absorbed by cells. Ligand absorption $(J)$ is investigated.

The model is reduced to a weakly coupled two component system in which the reaction term in each diffusion equation is monotone increasing in the other component. A strong comparison theorem is proved which leads to a uniqueness proof. Various limiting cases of the model are considered in which comparison theorems are repeatedly applied to obtain solution bounds and estimates. The experimentally observed behaviour of $J$ is shown to be a consequence of the interplay between volumetric processes (differential equations) and surface processes (boundary conditions) without recourse to invoking special cell-surface mechanisms for transferring the ligand from the binding protein into the cells.

\section{Other applications}

Pao[18] considers a steady-state formulation for the transport process of $M$ types of particles along a finite rod. Particles move in both the forward and backwards 
direction along the rod. They are destroyed by collisions and formed by both particle interactions and internal sources.

Existence and uniqueness of non-negative solutions are studied using the method of successive approximations. A recursion formula is derived for the calculation of the maximal and minimal solutions which are the limits of monotonically nonincreasing and monotonically nondecreasing sequences respectively. Conditions under which the maximal and minimal solutions coincide, thus uniqueness, are proved. The existence and nonexistence of a nonnegative solution is shown to be characterised by the convergence or nonconvergence, respectively, of the minimal sequence which is obtained from the recursion formula with zero initial iteration.

Lee and Hill[10] consider a three-component system of parabolic partial differential equations describing diffusion in the presence of three diffusion paths. The

three reaction functions $f_{i}$ are linear: $f_{i}=\sum_{j=1}^{3} a_{i j} u_{j}$. Sufficient conditions on the constants $a_{i j}$ are found which guarantee maximum principles for the system, i.e. conditions so that at least one of the diffusing species attains its maximum on $\partial B$.

\section{Theoretical developments not motivated by application}

Kastenberg[8] considers the $M$-component problem $(s=1, \ldots, M)$

$$
\begin{aligned}
\frac{\partial u_{s}(\mathbf{x}, t)}{\partial t} & =L_{s}(\mathbf{x}) u_{s}(\mathbf{x}, t)+\mathcal{F}_{s}\left(\mathbf{x}, t, u_{1}, \ldots, u_{M}\right), \\
u_{s}(\mathbf{x}, t) & =0, \mathbf{x} \in \partial B
\end{aligned}
$$

where the $L_{s}$ are uniformly elliptic operators of the form

$$
L_{s}(\mathbf{x}) \equiv \sum_{i, j=1}^{n} a_{i j}^{s}(\mathbf{x}) \frac{\partial^{2}}{\partial x_{i} \partial x_{j}}+\sum_{j=1}^{n} b_{j}^{s}(\mathbf{x}) \frac{\partial u}{\partial x_{i}}+c^{s}(\mathbf{x}), \mathbf{x} \in B,
$$

and the functions $\mathcal{F}_{s}$ have the general form

$$
\mathcal{F}_{s}\left(\mathbf{x}, t, u_{1}, \ldots, u_{M}\right) \equiv \sum_{k=1}^{K} \int_{0}^{t} \mathrm{~d} t^{\prime} \int_{x} \mathrm{~d} \mathbf{x}^{\prime} \mathcal{H}_{K}\left[\mathbf{x}, t ; \mathbf{x}^{\prime}, t^{\prime}, u_{1}(\mathbf{x}, t), \ldots, u_{M}\left(\mathbf{x}^{\prime}, t^{\prime}\right)\right] .
$$

Let $G_{s}[\mathbf{x}, t, V]$ represent a comparison system defined by

$$
G_{s}[\mathbf{x}, t, V] \equiv L_{s}(\mathbf{x}) v_{s}(\mathbf{x}, t)+\mathcal{G}_{s}\left(\mathbf{x}, t, v_{1}, \ldots, v_{M}\right)
$$

with a nonlinearity of the same form as equation (36). Weak and strong comparison theorems are proved which do not restrict $\mathcal{F}_{s}\left(\mathbf{x}, t, u_{1}, \ldots, u_{M}\right)$ to be a positive monotone function: It can vary in sign as well as slope. The weak form requires $\mathcal{G}_{s}$ to satisfy a uniform Lipschitz condition.

Dhaigude and Kasture[5] prove strong and weak comparison theorems concerning the Dirichlet problem for a class of weakly coupled nonlinear second order elliptic 
systems. Some simple applications of the weak comparison theorem to problems on deflection or vibration of plates and membranes are discussed.

Howes[7] studies the stability of steady-state solutions of the perturbed multicomponent reaction-diffusion system

$$
u_{t}+f(\mathbf{x}, u)=\epsilon^{2} u_{x x},
$$

in a rectangle in $\Re^{2}$ satisfying either Dirichlet or Robin boundary conditions. The perturbation parameter $\epsilon^{2}$ is assumed to be small. An essentially linear stability analysis is followed by a nonlinear stability analysis that shows how the nonlinear terms modify the linear result and provide sharp bounds on the domain of asymptotic stability of the steady solution under study. These results are proved using a comparison theorem that establishes bounds on the growth/decay of perturbations to the steady solution.

\section{Discussion}

In this paper we have discussed developments in the theory and applications of comparison theorems, as traced through citations of one of the first papers to be published in this area. Naturally such a review can not claim to be a comprehensive treatment of the subject. Even so, we hope to have shown that there has been a successful synergy between theoretical developments and practical motivation.

The power of comparison theorems is that sub- and super-solutions provide solution bounds and that it is considerably easier to solve inequalities rather than equalities. Comparison theorem techniques have important applications beyond existence and uniqueness proofs and are a useful tool for any applied mathematician working in reaction-diffusion systems to have in their armoury.

One such application which springs to mind is the investigation into the cellular uptake of ligands considered by McNabb and Bass[14]. Here the model problem involves wide classes of surfaces with arbitrarily complicated shapes, comparison theorems provide precise results of general importance which could not be achieved by detailed computation.

In 1961 restrictive monotonicity requirements on reaction functions were required. Pachpatte[16] has proved the existence and uniqueness of general systems of coupled reaction-diffusion equations without imposing monotonicity conditions on the reaction functions. Pachpatte's approach is based on rewriting the problem as a system of Volterra-Fredholm type integral equations by means of a Green's function representation and then applying the Banach fixed point theorem. However, as comparison theorems have many applications outside of existence and uniqueness proofs it is desirable to have comparison theorems for non-monotone systems.

In[13] it is shown that any non-monotone weakly coupled system can be embedded in a monotone weakly coupled system of twice the order. Since every solution of the first system is a solution of the higher order one, bounds can be obtained in the higher order monotone system. This embedding result remains little known and its 
power has yet to be fully appreciated. In addition to the embedding technique this paper includes a study of comparison theorems for ordinary differential equations.

\section{Acknowledgments}

This work was carried out whilst I was supported by the EPSRC (GR/L28142). During it I benefited from frequent discussions with Alec McNabb. This paper would have been impossible without his contributions.

\section{References}

1. N.F. Britton. Reaction-Diffusion Equations and Their Applications to Biology. AcademicPress, 1st edition, 1986.

2. V. Capasso. Asymptotic Stability for an Integrodifferential Reaction-Diffusion System. Journal of Mathematical Analysis and Applications, 103(2):575-588, 1984.

3. P.J. Chen, R.R. Nachlinger, and J.W. Nunziato. A Uniqueness Theorem for Nonlinear Heat Conduction in Chemically Reacting Media. Journal of Mathematical Analysis and Applications, 53:137-144, 1976.

4. P.J. Chen and J.W. Nunziato. Linear Theory of Heat Conduction in Chemically Reacting Media. Acta Mechanica, 22:267-280, 1975.

5. D.B. Dhaigude and D.Y. Kasture. Comparison Theorems for Nonlinear Elliptic Systems of Second Order with Applications. Journal of Mathematical Analysis and Applications, 112:178-189, 1985.

6. A. Friedman. Partial Differential Equations of Parabolic Type. Prentice-Hall, Inc., 1st edition, 1964.

7. F.A. Howes. Stability of Steady Solutions of Reaction-Diffusion Equations. Nonlinear Analysis, Theory, Methods \& Applications, 10(11):1209-1221, 1986.

8. W.E. Kastenberg. Comparison Theorems for Nonlinear Multicomponent Diffusion Systems. Journal of Mathematical Analysis and Applications, 29:299-304, 1970.

9. T. Kusano. On the Cauchy Problem for a Class of Multicomponent Diffusion Systems. Proceedings of the Japan Academy, 39(9):634-638, November 1963.

10. A.I. Lee and J.M. Hill. On Maximum Principles for Diffusion in the Presence of Three Diffusion Paths. Journal of the Australian Mathematical Society Series B - Applied Mathematics, 24:417-423, April 1983.

11. V.N. Maslennikova and N.V. Gapeeva. Problem Involving a Directional Derivative for Multicomponent Diffusion Systems and Chemical Kinetics. Siberian Mathematical Journal, 27:797-804, 1980. Translated from Sibirskii Mathematicheskii Zhurnal, Vol 21, No. 6, pp. 61-70, November-December, 1980.

12. A. McNabb. Comparison and existence theorems for multicomponent diffusion systems. Journal of Mathematical Analysis and Applications, 3:133-144, 1961.

13. A. McNabb. Comparison Theorems for Differential Equations. Journal of Mathematical Analysis and Applications, 119(1/2):417-428, October/November 1986.

14. A. McNabb and L. Bass. A Diffusion-Reaction Model for the Cellular Uptake of ProteinBound Ligands. SIAM Journal of Applied Mathematics, 51(1):124-149, February 1991.

15. Jia-Qi Mo. Singular Perturbation For a Class of Nonlinear Reaction Diffusion Systems. Science in China (Series A), 32(11):1306-1315, November 1989.

16. B.G. Pachpatte. On Nonlinear Coupled Reaction-Diffusion Equations. Applied Mathematics and Computation, 16(4):297-307, 1985.

17. C.V. Pao. Positive Solution of a Nonlinear Diffusion System Arising in Chemical Reactors. Journal of Mathematical Analysis and Applications, 46:820-835, 1974. 
18. C.V. Pao. An iterative method for solving a two-point boundary-value problem. Journal of Mathematical Physics, 16(5):1134-1138, May 1975.

19. C.V. Pao. Asymptotic Stability of Reaction-Diffusion Systems in Chemical Reactor and Combustion Theory. Journal of Mathematical Analysis and Applications, 82:503-526, 1981.

20. A. Parshotam, A. McNabb, and G. Wake. Comparison Theorems for Couple ReactionDiffusion Equations in Chemical Reactor Analysis. Journal of Mathematical Analysis and Applications, 178:196-220, 1993.

21. M.H. Protter and H.F. Weinberger. Maximum Principles in Differential Equations. PrenticeHall Partial Differential Equation Series. Prentice-Hall, Inc, 1st edition, 1967.

22. J. Smoller. Shock Waves and Reaction-Diffusion Equations. Grundlehren der mathematischen Wissenschaften 258. Springer-Verlag, 2nd edition, 1994.

23. R. Sperb. Maximum Principles and Their Applications, volume 157 of Mathematics in Science and Engineering. Academic Press, 1st edition, 1981.

24. G.C. Wake. On Comparison Theorems for Multicomponent Diffusion Systems. Journal of Mathematical Analysis and Applications, 26:292-296, 1969.

25. G.C. Wake. Non-linear heat generation with reactant consumption. The Quarterly Journal of Mathematics, 22(88):583-95, December 1971.

26. S.A. Willians and P-L Chow. Nonlinear Reaction-Diffusion Models for Interacting Populations. Journal of Mathematical Analysis and Applications, 62:157-169, 1978. 


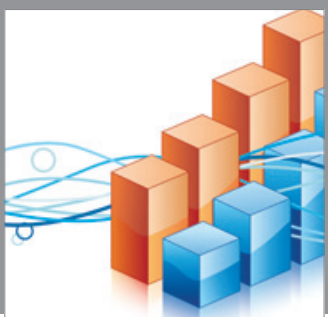

Advances in

Operations Research

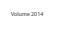

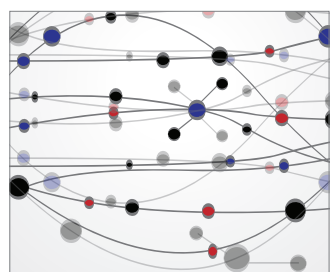

\section{The Scientific} World Journal
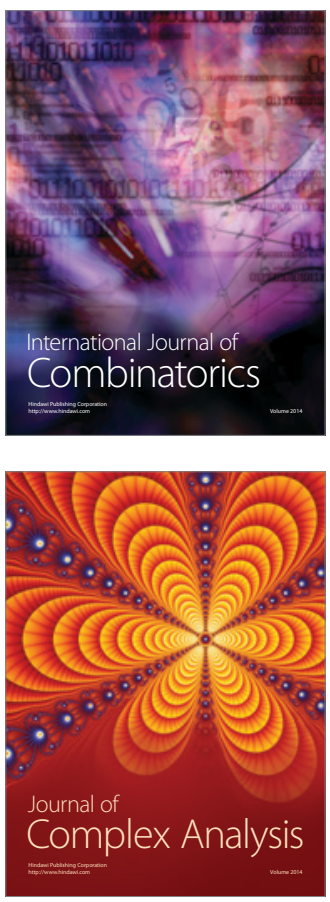

International Journal of

Mathematics and

Mathematical

Sciences
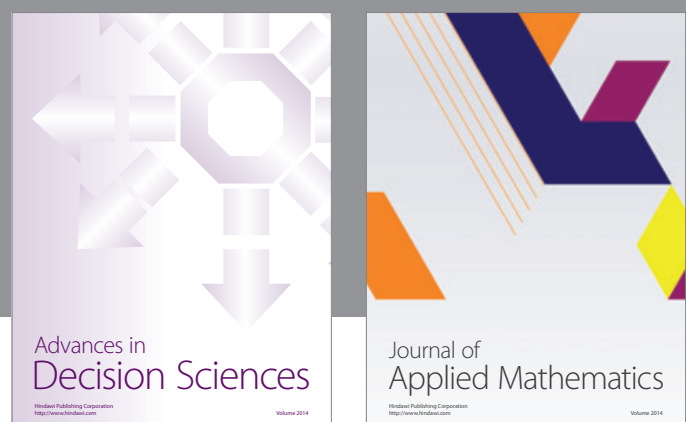

Journal of

Applied Mathematics
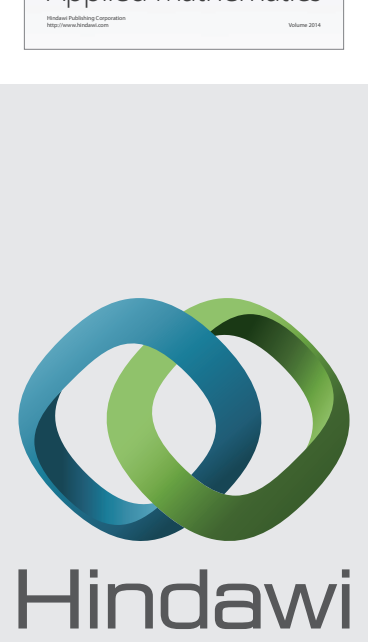

Submit your manuscripts at http://www.hindawi.com
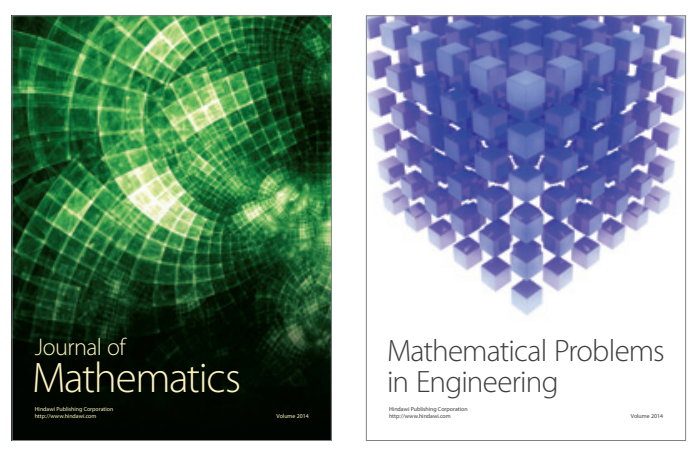

Mathematical Problems in Engineering
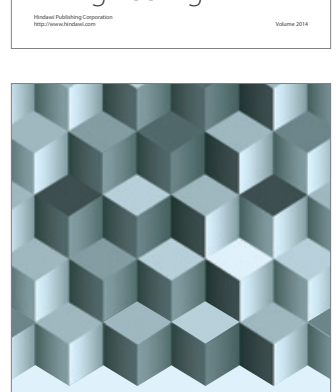

Journal of

Function Spaces
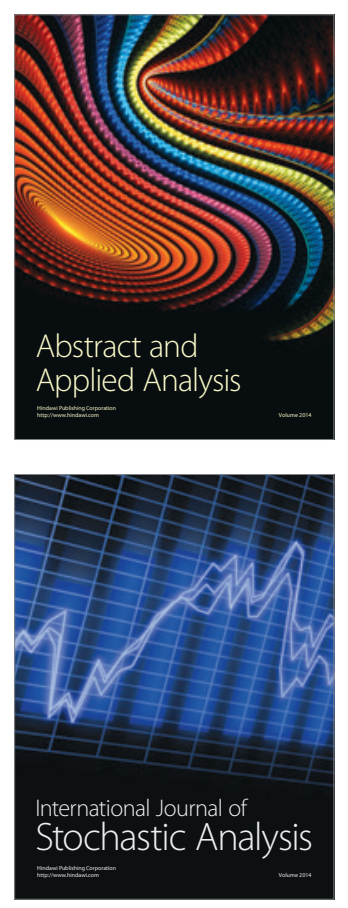

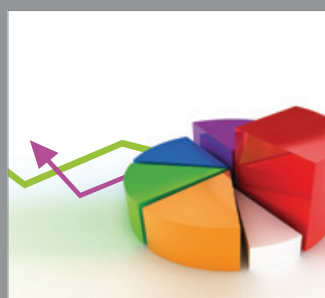

ournal of

Probability and Statistics

Promensencen
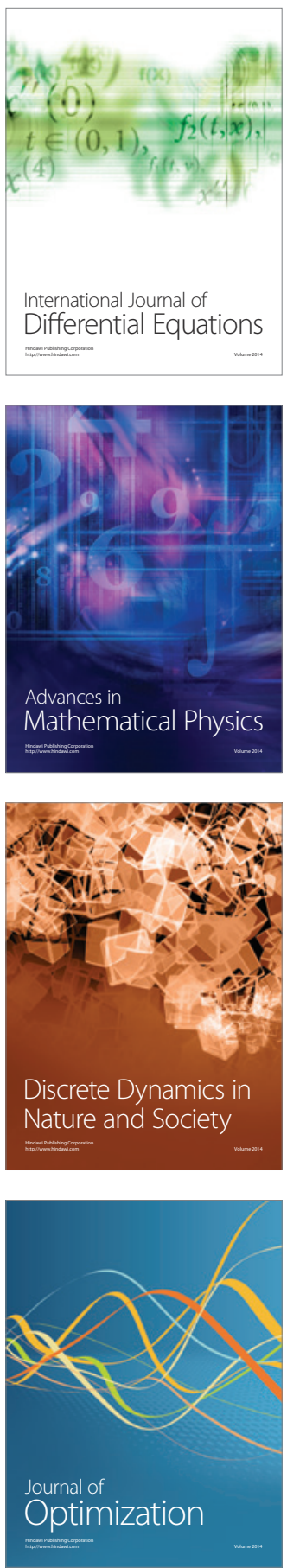